\title{
Gait Ignition Failure in JNPL3 Human Tau-mutant Mice
}

\author{
HoChung Jang ${ }^{1}$, Jung Hwa Ryu ${ }^{1}$, Kyung Min Shin ${ }^{1}$, Na-young Seo ${ }^{1,2}$, \\ Gyu Hyun Kim ${ }^{1}$, Yang Hoon $\mathrm{Huh}^{3}$, Ae Nim Pae ${ }^{4}$ and Kea Joo Lee ${ }^{1,2 *}$ \\ ${ }^{1}$ Department of Structure and Function of Neural Network, Korea Brain Research Institute, Daegu 41062, \\ ${ }^{2}$ Department of Brain and Cognitive Sciences, DGIST, Daegu 42988, ${ }^{3}$ Center for Electron Microscopy Research, \\ Korea Basic Science Institute, Cheongju 28119, ${ }^{4}$ Convergence Research Center for Diagnosis, Treatment and \\ Care System of Dementia, Korea Institute of Science and Technology, Seoul 02792, Korea
}

\begin{abstract}
Cognitive impairments and motor dysfunction are commonly observed behavioral phenotypes in genetic animal models of neurodegenerative diseases. JNPL3 transgenic mice expressing human P301L-mutant tau display motor disturbances with age- and gene dose-dependent development of neurofibrillary tangles, suggesting that tau pathology causes neurodegeneration associated with motor behavioral abnormalities. Although gait ignition failure (GIF), a syndrome marked by difficulty in initiating locomotion, has been described in patients with certain forms of tauopathies, transgenic mouse models mirroring human GIF syndrome have yet to be reported. Using the open field and balance beam tests, here we discovered that JNPL3 homozygous mice exhibit a marked delay of movement initiation. The elevated plus maze excluded the possibility that hesitation to start in JNPL3 mice was caused by enhanced levels of anxiety. Considering the normal gait ignition in rTg4510 mice expressing the same mutant tau in the forebrain, GIF in JNPL3 mice seems to arise from abnormal tau deposition in the hindbrain areas involved in locomotor initiation. Accordingly, immunohistochemistry revealed highly phosphorylated paired helical filament tau in JNPL3 brainstem areas associated with gait initiation. Together, these findings demonstrate a novel behavioral phenotype of impaired gait initiation in JNPL3 mice and underscore the value of this mouse line as a tool to study the neural mechanisms and potential treatments for human GIF syndrome.
\end{abstract}

Key words: Tau, Gait ignition, Motor behavior, Neurodegenerative disease, Tauopathy

\section{INTRODUCTION}

Cognitive deficits and motor dysfunction are frequently observed behavioral phenotypes in genetic animal models of neurodegenerative diseases [1]. Neurofibrillary tangles (NFT) composed

Received April 10,2019, Revised May 19, 2019,

Accepted May 27, 2019

* To whom correspondence should be addressed. TEL: 82-53-980-0320, FAX: 82-53-980-8339 e-mail: relaylee@kbri.re.kr of highly phosphorylated forms of microtubule-associated protein tau are commonly found in various tauopathies such as Alzheimer's disease (AD) [2], Pick's disease [3], progressive supranuclear palsy [4], frontotemporal dementia [5], Parkinsonism linked to chromosome 17 (FTDP-17) [6], and corticobasal degeneration $[4,7]$, implicating tau dysfunction in the pathology underpinning neurodegeneration and behavioral deficits. Preceding the onset of neurodegeneration, hyperphosphorylated tau proteins undergo abnormal assembly, leading to the formation of diverse tau protein species [8]. These various mis-processed tau aggregates lead to cognitive and motor impairments, potentially by interrupting 
normal synaptic circuit function $[9,10]$. In JNPL3 transgenic (Tg) mice, expression of human tau containing the most common FTDP-17 mutation (P301L) under the control of the mouse prion promoter resulted in motor disturbances including weakened grasping strength, hunched posture, and hindlimb paralysis with age- and gene-dose-dependent development of NFT [11]. NFT and Pick-body-like inclusions were observed in the amygdala, septal nuclei, pre-optic nuclei, hypothalamus, midbrain, pons, medulla, deep cerebellar nuclei, and spinal cord of JNPL3 mice, with tau-immunoreactive pre-tangles observed in the cerebral cortex, hippocampus, and basal ganglia [11]. Therefore, better understanding of the behavioral traits associated with overexpressed mutant human tau is critical for the identification of new therapeutic strategies for neurodegenerative disorders.

Gait ignition failure (GIF) is a syndrome characterized by the hesitation or inability to initiate gait from a static position [12, 13]. Interestingly, once this hesitation is overcome, gait is normal; or at least resembles the patient's normal pattern [14]. Gait often arrests again during turns, while traversing narrow spaces such as doorways, or when distracted [12]. GIF phenotype is observed in several neurological disorders, including normal pressure hydrocephalus [15], subcortical vascular disease [16], parkinsonian syndromes [17], progressive supranuclear palsy [18], corticobasal degeneration [19], multiple system atrophy [20], and various focal lesions [21]. This indicates that GIF may occur in some forms of tauopathies. This led us to hypothesize that JNPL3 mice may display behavioral characteristics of GIF syndrome. Despite several clinical studies describing GIF syndrome in human patients [13, $14,16,19,21-24]$, the corresponding behavioral phenotype has not been reported in genetic mouse models of tauopathies.

Using a battery of behavioral tests including 1) the open-field test, 2) the balance beam task, 3) the coat hanger task, and 4) the elevated plus maze test, we demonstrate here that JNPL3 mice expressing Prion-promoter-driven human mutant tau exhibit a marked delay of gait initiation that recapitulates GIF syndrome in human neurodegenerative diseases. Their hesitation or delay initiating exploration is unlikely to be caused by enhanced anxiety because JNPL3 mice had similar anxiety levels in the elevated plus maze compared to wild-type (WT) animals. Intriguingly, rTg4510 mice expressing the same mutant tau driven by the CaMKII $\alpha$ promoter in the forebrain display normal gait ignition, suggesting that gait freezing in JNPL3 mice may result from abnormal tau deposition in the hindbrain areas associated with locomotion. In support of this idea, immunohistochemistry revealed highly phosphorylated paired helical filament (PHF) tau in the JNPL3 brain stem areas involved in gait initiation. Together, these findings demonstrate a novel behavioral phenotype of impaired gait ignition in JNPL3 mice and underscore the value of this mouse model as a tool to study the underlying mechanisms and potential treatments for human GIF syndrome.

\section{MATERIALS AND METHODS}

\section{Animals}

Homozygous JNPL3 Tg mice (Tg(Prnp-MAPTP301L)JNPL3Hlmc, stock number 2508) were purchased from Taconic Biosciences (Rensselaer, NY, USA). JNPL3 and age-matched WT mice were housed in polycarbonate cages with a 12-h light/dark cycle, and had access to food and water ad libitum. Animals were subjected to a series of behavioral assessments at 8 to 12 months of age. Additionally, 21-month-old mice were tested in the open field test. All animal experiments were performed in accordance with the Institutional Animal Care and Use Committee of the Korea Brain Research Institute (IACUC-17-00018).

\section{Behavioral tests}

Behavioral tests were performed during the light phase (08:00 to 17:00). Prior to each behavioral test, all subjects underwent 30 minutes of habituation in a sound-attenuated experimental room with different illumination conditions (dim red light; 2.35 lux or bright light; 161.5 lux). The apparatus was cleaned with 70\% ethanol $\left(\mathrm{v} / \mathrm{v}\right.$ in $\left.\mathrm{dH}_{2} \mathrm{O}\right)$ between each trial of the behavioral test. All experiments were recorded to evaluate behavioral patterns of mice using video tracking software SMART 3.0 (Harvard Apparatus, Holliston, MA, USA).

\section{Coat hanger task}

Strength and coordination were tested in the coat hanger test [25]. The mouse was suspended by its forepaws from the center of the horizontal portion of a wire coat hanger. The goal was to raise its hind legs to the bar, move to the end of the bar, and right itself onto the diagonal portion of the coat hanger. Mice were subjected to three trials ( 60 seconds/trial) separated by at least 10 minutes. The latency to fall off the wire and a success assessment score of $0 \sim 3$ were recorded based on behavioral performance.

\section{Balance beam task}

To evaluate general coordination, equilibrium, and balance, each mouse was placed on a stationary beam and released. Mice were placed on $3 \mathrm{~cm}$-thick balance beam and trained to transverse the beam from the starting point to the goal box. All mice were successively trained at gradually longer starting points (10,20, and $40 \mathrm{~cm}$ apart from the goal box) for $120 \mathrm{sec}$ per trial. On test day, mice were placed on the same beam $70 \mathrm{~cm}$ from the goal box and 
allowed to across the beam. The balance time was averaged from three successive trials separated by at least 10 minutes. Maximum trial length was 120 seconds. The latency to traverse or fall off the beam was recorded.

\section{Open field test}

The open field test is a widely used behavioral paradigm for evaluating locomotor activity and anxiety in laboratory rodents. Mice were placed in the center of an open field box $(50 \times 50 \times 30 \mathrm{~cm})$ and allowed to move freely in the arena for $5 \mathrm{~min}$. The entire open field arena was divided into 16 grids to analyze behavioral patterns of JNPL3 mice, including total distance traveled, time spent in the central zone, and latency to gait ignition. The open field test was performed under two different light intensities: bright (161.5 lux) or dim red light (2.35 lux).

\section{Elevated plus maze test}

The elevated plus maze was conducted to evaluate anxietyrelated behavior in JNPL3 mice. The apparatus was constructed of opaque plastic with two sets of perpendicular arms (two open arms: $25 \times 6 \mathrm{~cm}$ and two closed arms: $25 \times 6 \times 20 \mathrm{~cm}$ ) with a central platform $(6 \times 6 \mathrm{~cm})$. The maze was placed $40 \mathrm{~cm}$ above the floor. Mice were placed on the central platform of the maze with the head positioned towards an open arm and were allowed to explore the maze for $5 \mathrm{~min}$.

\section{High-speed video recording system for gait analysis}

Inter-limb coordination was recorded using a high-speed camera (200 frames/sec) equipped with a semi-automated kinematic gait analyzing system (MotoRater, TSE system, Homburg, Germany). To reduce aversion to walking along an unfamiliar apparatus, JNPL3 and WT mice underwent an adaptation phase before the test trial. On test day, all mice were placed on a transparent linear corridor of MotoRater and allowed to walk along the aisle. To evaluate gait patterns in detail, each paw contact was analyzed per frame, and paw contact interval was calculated using TSE motion high-speed video analysis software.

\section{Immunohistochemistry}

After completion of behavioral tests, all mice were anesthetized with $4 \%$ avertin ( $\mathrm{v} / \mathrm{v}$, in warm phosphate-buffered saline [PBS]) and perfused with cold PBS (pH 7.4, Thermo Fisher Scientific, Waltham, MA, USA). Each extracted brain was divided into two hemispheres. Hemispheres underwent post-fixation in $4 \%$ paraformaldehyde (Biosesang, Seongnam, Korea) at $4^{\circ} \mathrm{C}$ for $24 \mathrm{hrs}$. Fixed brains were cryo-protected in 30\% sucrose (in PBS) and frozen with optimal cutting temperature (OCT) compound. Brains were stored at $-80^{\circ} \mathrm{C}$ for subsequent immunohistochemistry. Premounted sagittal brain sections (30- $\mu \mathrm{m}$-thick) were incubated with permeabilization solution (0.3\% Triton X-100, 0.05\% Tween 20 in PBS) for 15 minutes, followed by blocking solution (5\% normal goat serum in PBS) for $1 \mathrm{hr}$. All sections were then incubated with primary antibody mixture, composed of PHF-tau (AT8) antibody (1:250, Invitrogen MN1020, Waltham, MA, USA) and NeuN antibody (1:300, Abcam ab190195, Cambridge, UK), diluted in antibody dilution solution (0.3\% Triton X-100, 2\% normal goat serum in PBS) at $4^{\circ} \mathrm{C}$ overnight. After three washes in PBS, sections were incubated with a mixture of corresponding fluorescent secondary antibodies for $2 \mathrm{hr}$ at room temperature. Immunostained sections were incubated with Hoechst staining solution (Sigma, St.Louis, MO, USA) for $10 \mathrm{~min}$. To reduce lipofuscin-derived autofluorescence, immunostained sections were incubated with $0.1 \%$ Sudan Black B (in 70\% EtOH, Sigma) for 20 min at room temperature. After completion of all staining processes, all sections were mounted with antifade mounting medium (Biomeda, Foster City, CA, USA).

\section{Statistical analysis}

All data are expressed as means \pm standard error of the mean. P values and all graphs were generated using GraphPad Prism 8. Statistical significance was calculated using two distinct statistical methods, including Mann-Whitney test and two-tailed unpaired $t$ test, depending on normal (Gaussian) distribution of data. All statistical analyses were carried out after conducting Grubbs outlier identification test. The level of statistical significance was set at $\mathrm{p}<0.05$.

\section{RESULTS}

JNPL3 Tg mice expressing human P301L mutant tau protein exhibit progressive motor disturbances related to development of NFT, including weakened grasping strength and hindlimb paralysis [11]. To validate the pathologic development of motor deficits in JNPL3 homozygous mice, we initially performed the coat hanger and balance beam walk tests. At 8 months of age, JNPL3 mice fell more quickly from the coat hanger wire when compared to WT animals (Fig. 1A C) (Latency to fall [in sec]: WT, 44.6 \pm 7.6 ; JNPL3, 4.9 $\pm 0.9 ; \mathrm{p}=0.0006$; Coat hanger score: WT, $1.43 \pm 0.48$; JNPL3, 0; $\mathrm{p}=0.021$ ), indicating weakened paw strength and impaired motor coordination. Similarly, aged JNPL3 (12-monthold) mice performed significantly worse on the balance beam test than did WT animals (Fig. 1D F) (Latency to cage [in sec]: WT, 20.5 $\pm 8.5 ;$ JNPL3, $120.0 \pm 0 ; \mathrm{p}<0.0001$; Latency to fall [in sec]: WT, 111.4 $\pm 8.6 ;$ JNPL3, 31.2 $\pm 7.3 ; \mathrm{p}<0.0001$ ). 
A

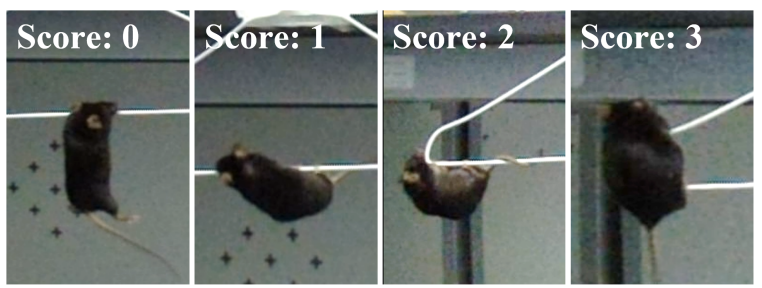

D

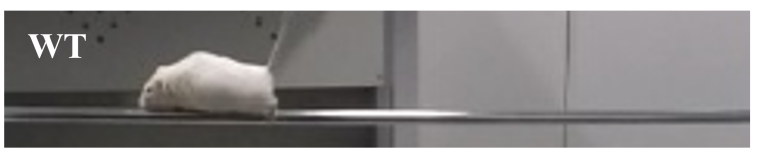

\section{JNPL3}

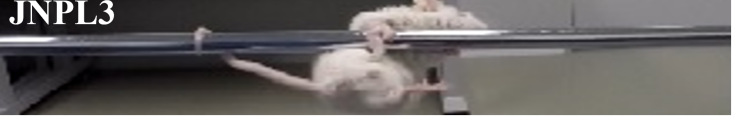

B

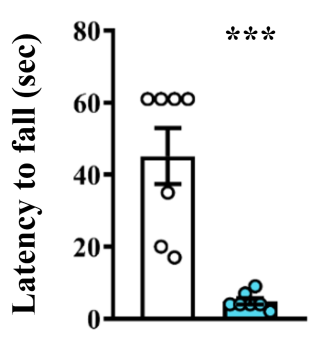

E

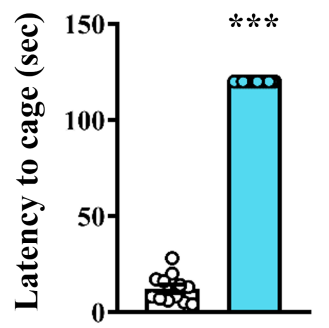

C

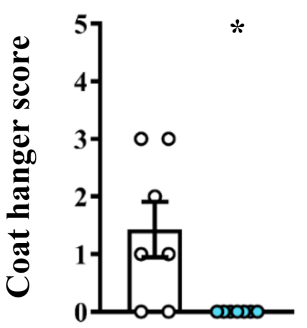

F

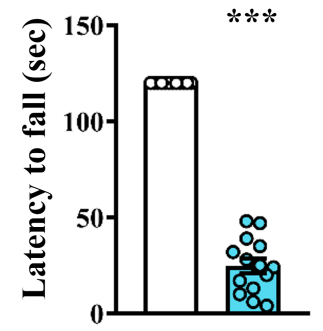

G

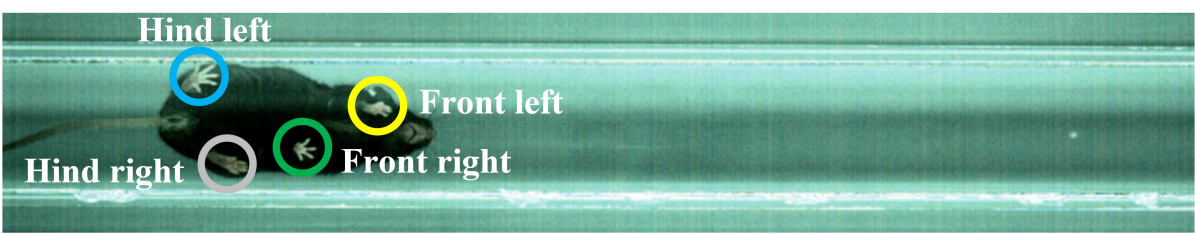

H

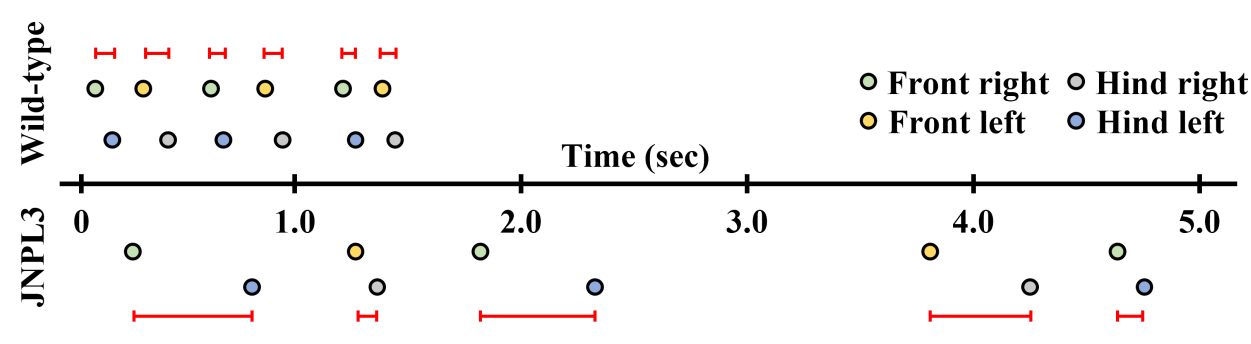

I

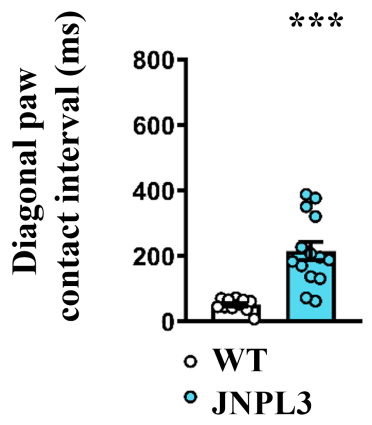

Fig. 1. Impaired motor coordination in JNPL3 mice. (A C) Coat hanger test to evaluate limb strength and coordination (WT, $\mathrm{n}=7$; JNPL3, $\mathrm{n}=7$, 8-month-old). (A) Success assessment scoring and representative image of each score. (B) Latency to fall off the wire in WT and homozygous JNPL3 mice. (C) Appraised scores based on scoring criteria in (A). Mann-Whitney test, ${ }^{*} \mathrm{p}<0.05,{ }^{\star * *} \mathrm{p}<0.001$. (D F) Goal-directed balance beam test to evaluate general coordination and balance (WT, $n=13$; JNPL3, $n=15,12$-month-old). (D) Representative image comparing motor performance between WT and JNPL3 mice. (E) Time spent to cross the balance beam. (F) Latency to fall off the beam. Mann-Whitney test, ${ }^{* * *} \mathrm{p}<0.001$. (G I) Trotting gait pattern analysis of WT and JNPL3 mice (WT, n=12; JNPL3, n=15, 12-month-old). (G) Representative paw contact image of ground walking obtained from high-speed video recording system. (H) Four-paw contact points over time displaying gait pattern. (I) Diagonal paw contact interval indicating limb coordination in WT and JNPL3 mice. Two-tailed unpaired t test, ${ }^{* * *} \mathrm{p}<0.001$.

Based on the observed motor dysfunction in JNPL3 mice, we reasoned that JNPL3 mice would display aberrant walking patterns. To examine this, we performed kinematic gait analysis in ground walking using a high-speed video tracking system. The analysis of gait patterns revealed that 12-month-old JNPL3 mice had significantly longer intervals between diagonal paw contacts when compared to WT animals (Fig. 1G I; Video S1) (Diagonal paw contact interval [in msec]:WT, 65.4 \pm 14.6 ; JNPL3, 24 3.0 \pm 38.5 ; $\mathrm{p}<0.0001$ ), indicating that JNPL3 mice developed severe disintegration of interlimb coordination. In addition, we observed that most JNPL3 mice presented with forelimb tremors and obtundation of hindlimbs caused by paralysis, consistent with a previous 
report [11]. These results suggest that JNPL3 homozygous mice have severe motor deficits in locomotor activity.

During the balance beam test, we noticed that JNPL3 mice tended to take longer to initiate movement, resembling the GIF phenotype in human neurodegenerative diseases $[13,14]$. To examine whether JNPL3 mice displayed behavioral phenotypes

Open-field test (12-month-old)

A

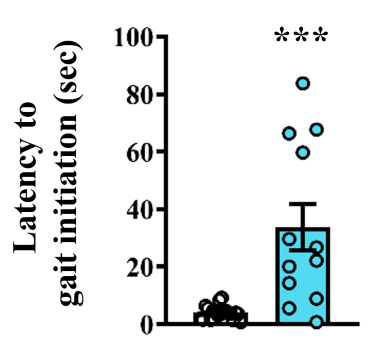

D

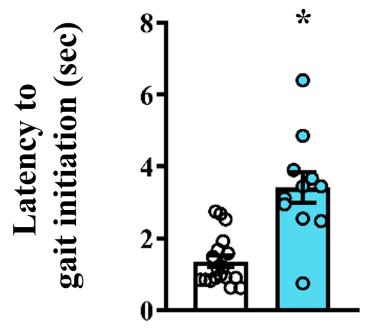

B

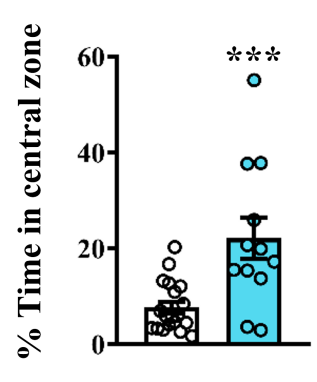

E

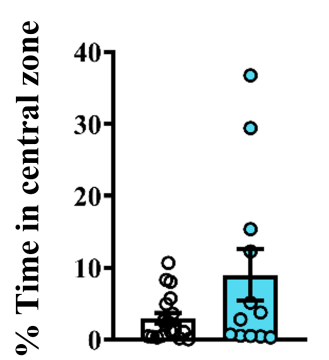

C

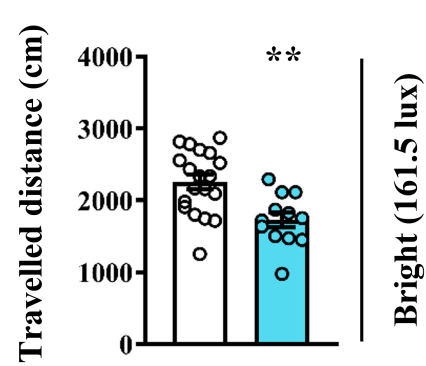

$\mathbf{F}$

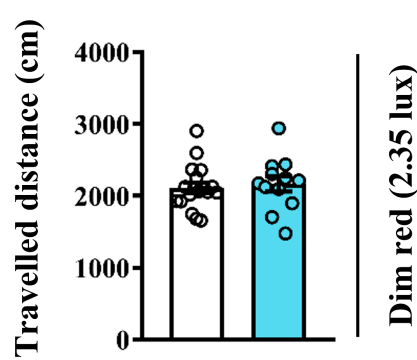

Open-field test (21-month-old)

G

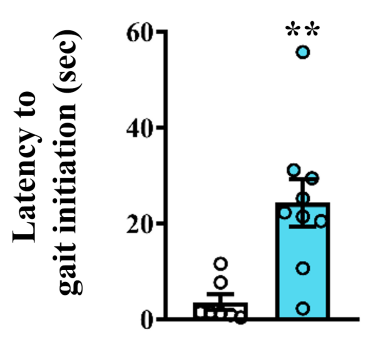

H

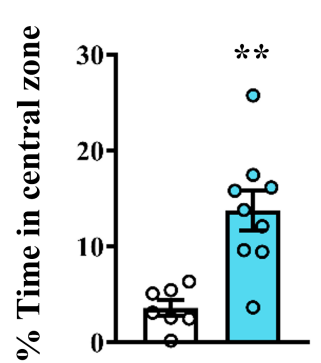

I

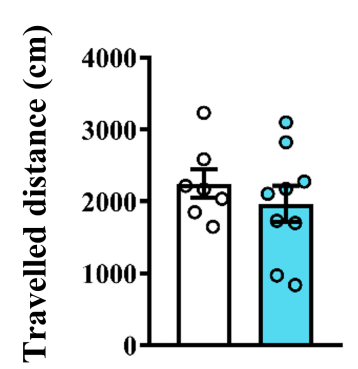

Balance beam test

$\mathbf{J}$

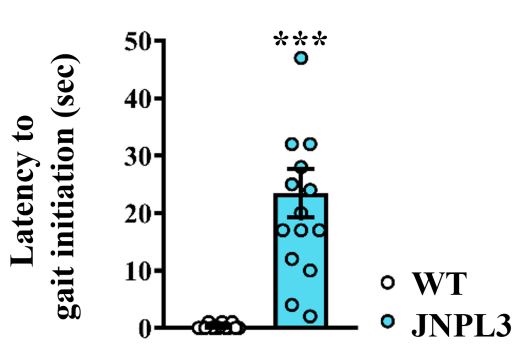

\section{Open-field test}

K

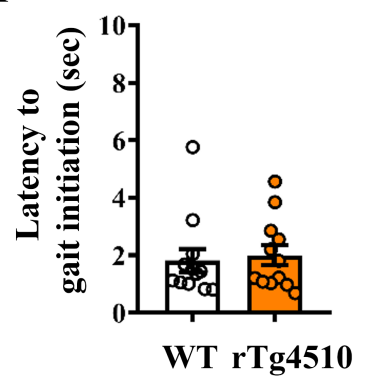

Fig. 2. Delayed gait ignition in JNPL3 mice. (A F) Open-field test to evaluate general locomotor activity in 12-month-old WT and JNPL3 mice under bright $(\mathrm{A} \sim \mathrm{C})$ and dim red light $(\mathrm{D} \sim \mathrm{F})$ conditions (WT, $\mathrm{n}=19$; JNPL3, $\mathrm{n}=12$ ). Latency to gait ignition (A and D), time spent in the central zone of openfield arena (B and E), and total traveled distance (C and F) in WT and JNPL3 mice. Two-tailed unpaired $\mathrm{t}$ test, ${ }^{*} \mathrm{p}<0.05,{ }^{* *} \mathrm{p}<0.01,{ }^{* * *} \mathrm{p}<0.001 .(\mathrm{G} \sim \mathrm{I})$ Open-field test in 21-month-old WT and JNPL3 mice under bright light condition (WT, n=7; JNPL3, n=9). Mann-Whitney test, ${ }^{* *} \mathrm{p}<0.01$. (J) Gait initiation failure in balance beam walk test (WT, $\mathrm{n}=13$; JNPL3, $\mathrm{n}=15$ ). Mann-Whitney test, ${ }^{* * *} \mathrm{p}<0.001$. (K) Open-field test in 7-month-old WT and rTg4510 mice under bright light condition (WT, $\mathrm{n}=12 ; \mathrm{rTg} 4510, \mathrm{n}=12$ ). Two-tailed unpaired $t$ test, $\mathrm{p}>0.05$. 
mirroring human GIF syndrome, we performed the open-field test to assess exploratory behaviors and general locomotion. The level of illumination is a key factor that may affect anxiety and exploratory behavior in rodents [26]. Thus, we conducted the openfield test under two different illumination conditions: (1) bright illumination condition (Fig. 2A C); (2) dim red lighting condition (Fig. 2D F). In this test, WT mice initiated walking immediately after they were placed in the center zone of the open field arena. Notably, 12-month-old JNPL3 mice exhibited a significant delay in the onset of gait initiation regardless of lighting intensity (Fig. 2A and 2D; Video S2) (Latency to gait initiation under bright light [in sec]: WT, 4.0 0 0.5; JNPL3, 33.8 $\pm 8.1 ; \mathrm{p}<0.0001$; Latency to gait initiation under dim red light [in sec]: WT, $1.5 \pm 0.2$; JNPL3, 5.0 \pm 1.6 ; $\mathrm{p}=0.013$ ), although the difference in delay time between genotypes was modest in the dim light condition. JNPL3 mice spent more time in the central zone under bright illumination but not under dim red light (Fig. 2B and 2E) (Percentage of time in central zone under bright light: WT, 7.7 \pm 1.2 ; JNPL3, 22.1 \pm 3.3 ; $\mathrm{p}=0.0006$; Percentage of time in central zone under dim red light: WT, 3.9 \pm 1.2 ; JNPL3, 9.0 $\pm 3.6 ; \mathrm{p}=0.12$ ). Accordingly, under bright illumination, JNPL3 mice exhibited significantly decreased total exploratory distance relative to that of their WT littermates (Fig. 2C) (Distance travelled [in cm]: WT, 2254 \pm 102 ; JNPL3, 1727 $\pm 103 ; \mathrm{p}=0.0017$ ). In contrast, the total distance traveled by JNPL3 mice under dim light conditions was comparable to that of WT animals (Fig. 2F) (Distance travelled [in cm]: WT, 2196 \pm 107 ; JNPL3, 2168 \pm 107 ; p=0.86), which is reminiscent of GIF patients with normal gait patterns once the hesitation is overcome [14]. These findings suggest that the total amount of exploratory behavior in JNPL3 mice is differentially affected by different light intensities. In addition, the delayed gait ignition in the open field test was maintained in JNPL3 mice up to at least 21 months of age (Fig. 2G I) (Latency to gait initiation under bright light [in sec]: WT, 3.5 \pm 1.6 ; JNPL3, 24.3 \pm 5.0 ; $\mathrm{p}=0.0032$; Percentage of time in central zone under bright light: WT, 3.6 \pm 0.8 ; JNPL3, 17.5 $\pm 2.1 ; \mathrm{p}=0.001$; Distance travelled under bright light [in cm]: WT, 2248 \pm 198 ; JNPL3, 1968 \pm 252 ; $\mathrm{p}=0.42$ ). Importantly, the increased latency of gait initiation in JNPL3 mice was reproducible on the balance beam test, which requires goaldirected activity (Fig. 2J) (Latency to gait initiation [in sec]: WT, $0.2 \pm 0.1 ;$ JNPL3, 23.5 $\pm 4.2 ; \mathrm{p}<0.0001)$.

Based on these results, we conjectured that the heightened levels of anxiety in JNPL3 mice may have caused the delayed gait ignition in the open field and balance beam tests. Therefore, we conducted the elevated plus maze test to evaluate anxiety-like behavior in 12-month-old JNPL3 mice. Behavioral analysis revealed that JNPL3 mice had similar levels of anxiety compared to their WT littermates under both bright and dim light conditions (Fig. 3A B) (Number of total arm entries under bright light: WT, 9.3 \pm 1.4 ; JNPL3, 7.7 $\pm 1.6 ; \mathrm{p}=0.46$; Number of total arm entries under dim red light: WT, 13.6 \pm 1.4 ; JNPL3, 11.0 $\pm 1.9 ; \mathrm{p}=0.29$; Percentage of open arm entries under bright light:WT, 7.6 \pm 2.9 ; JNPL3, 16.0 \pm 5.4 ; $\mathrm{p}=0.15$; Percentage of open arm entries under dim red light: WT, 12.6 \pm 3.0 ; JNPL3, 22.1 $\pm 6.2 ; p=0.13$ ), suggesting that enhanced anxiety was unlikely to have induced the GIF phenotype in JNPL3 mice. Together, these findings suggest that the prolonged time taken to initiate spontaneous walking in JNPL3 mice is caused by a bona fide motor system dysfunction but not enhanced anxiety.

We next tested whether the GIF phenotype in JNPL3 mice resulted from tau P301L mutation per se using an independent tau transgenic mouse line, rTg4510, which expresses the same P301L mutant tau specifically in the forebrain driven by the CaMKIIa promoter [27, 28]. Seven-month-old rTg4510 mice have been

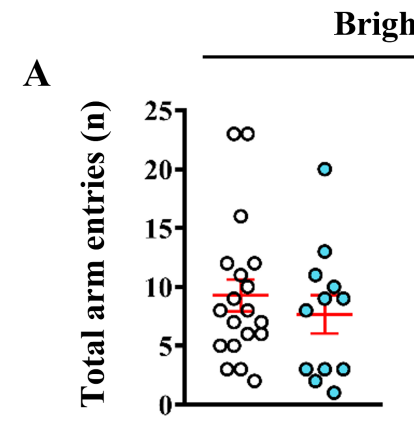

Bright (161.5 lux)

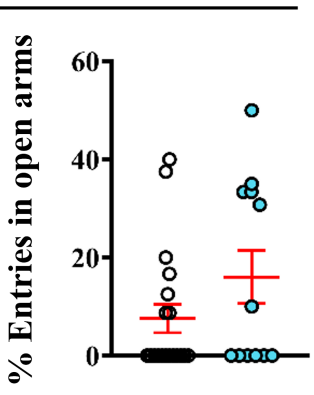

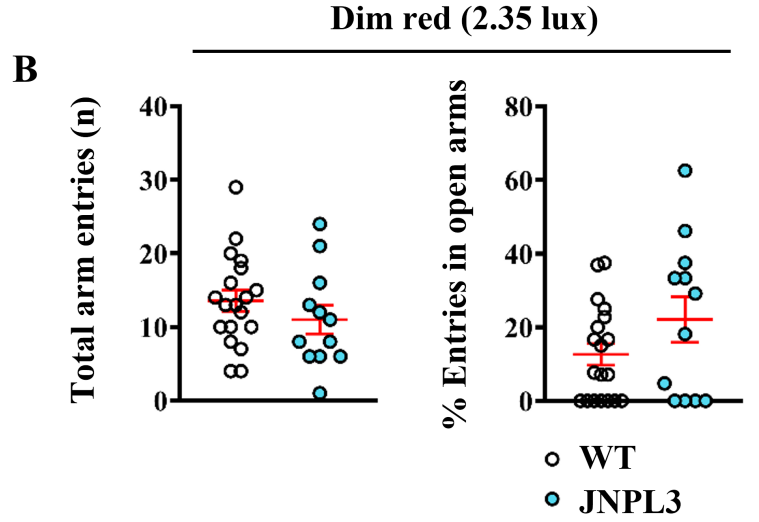

Fig. 3. Anxiety levels in JNPL3 mice. (A and B) The elevated plus maze test comparing anxiety-like behavior between WT and JNPL3 mice ( $\mathrm{n}=19$ and 12, respectively). The number of total arm entries representing exploratory activity (left) and proportion of entries into open arms (right) indicating anxiety levels in bright (A) and dim red light (B) conditions. Note that JNPL3 mice exhibited similar anxiety levels when compared to WT animals. Twotailed unpaired $t$ test, $\mathrm{p}>0.05$. 
A

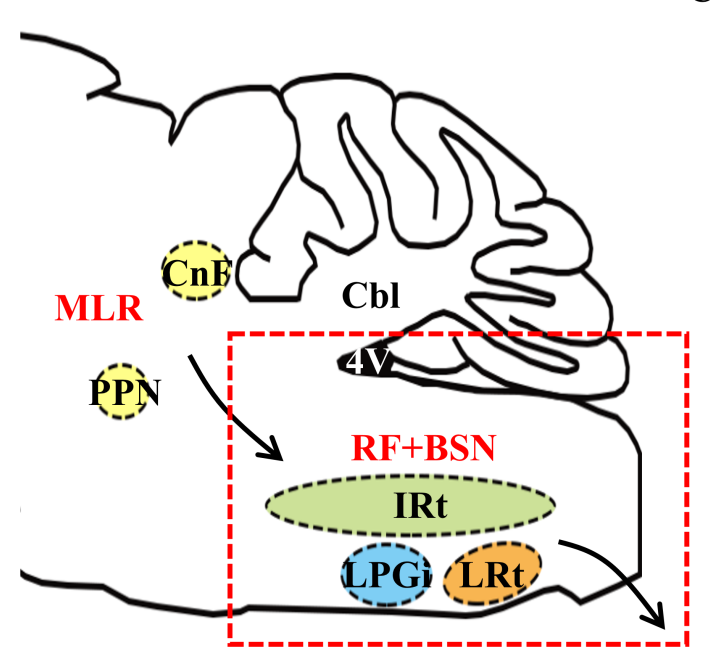

C
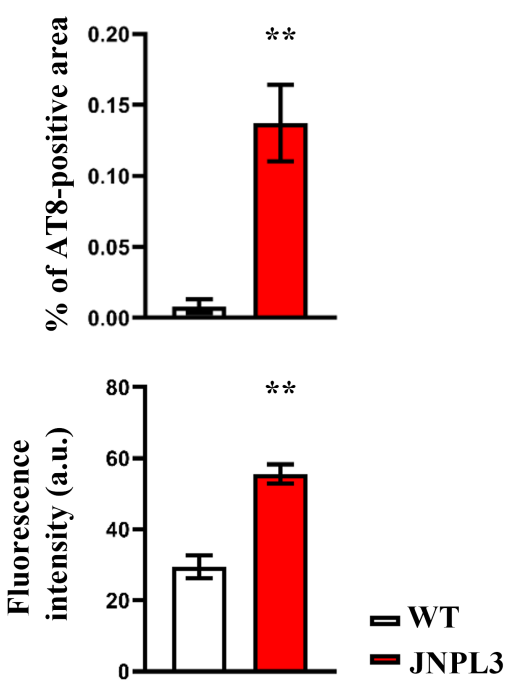

B

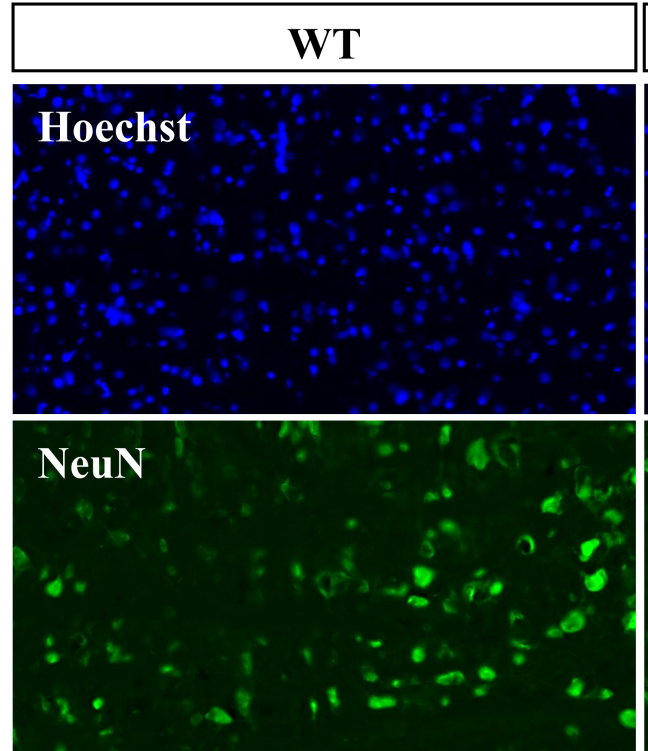

\section{PHF tau (AT8)}
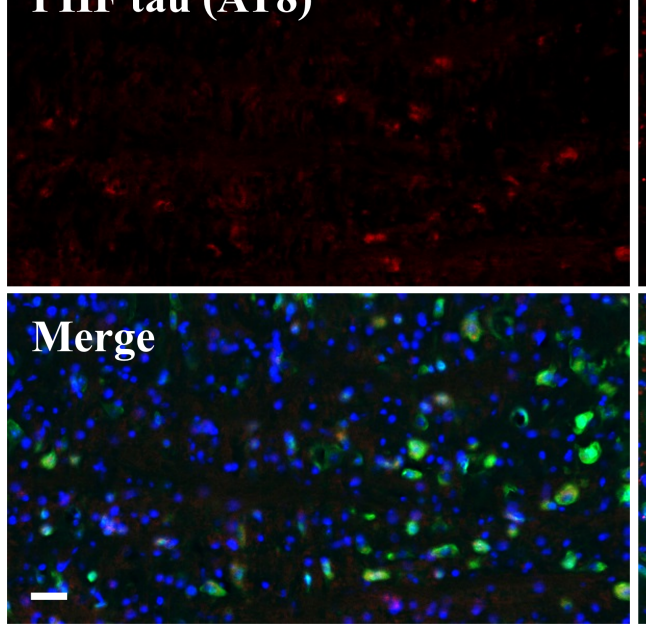
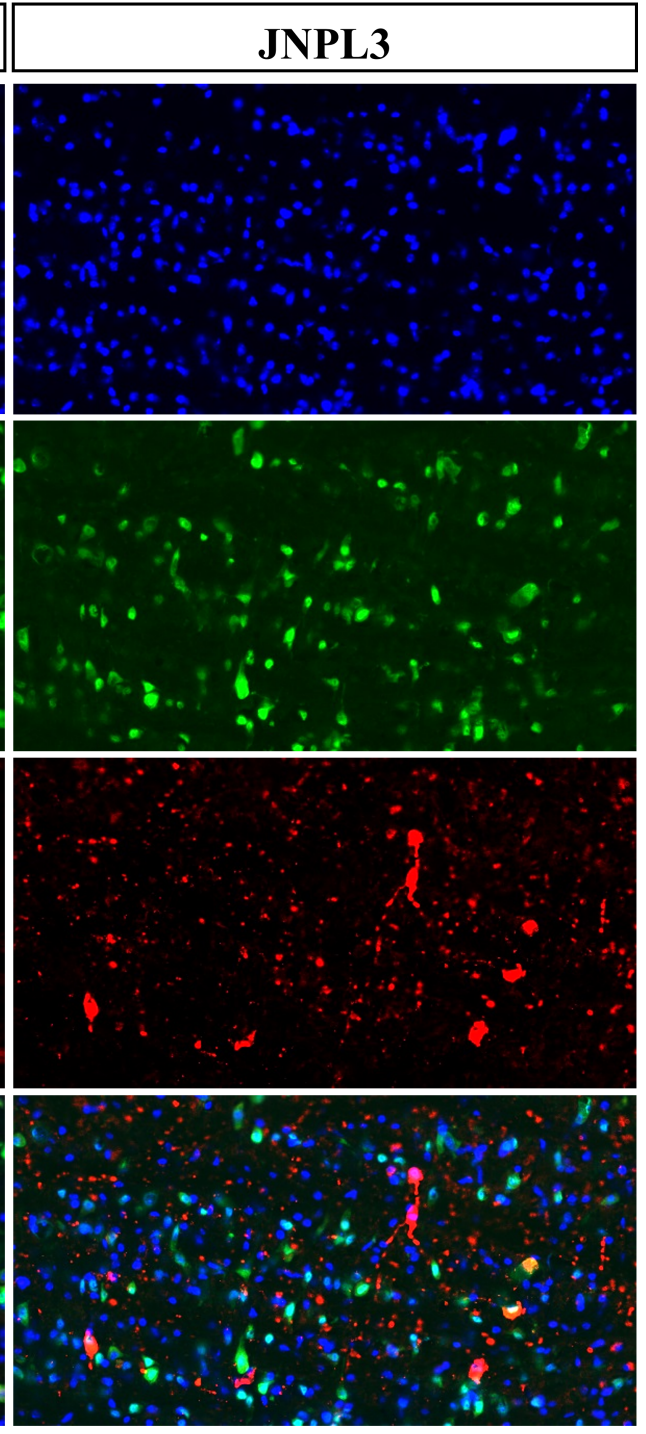

Fig. 4. Immunohistochemistry for PHF tau in JNPL3 hindbrain. (A) Schematic of circuits associated with initiation of locomotion (Cbl, cerebellum; MLR, mesencephalic locomotor region; $\mathrm{CnF}$, cuneiform nucleus; PPN, pedunculopontine nucleus; $\mathrm{RF}$, reticular formation; BSN, brainstem nuclei; IRt, Intermediate reticular nucleus; LPGi, Lateral paragigantocellular nucleus; LRt, Lateral reticular nucleus). (B) Immunohistochemical analysis of PHF tau expression levels in brainstem regions of JNPL3 mice (WT, n=3; JNPL3, n=3; 4V, fourth ventricle). Yellow boxes indicate magnified regions (LPGi). Scale bars, $50 \mu \mathrm{m}$. (C) Quantified PHF tau-immunopositive fluorescence intensity. Two-tailed unpaired $t$ test, ${ }^{*} \mathrm{p}<0.01$. 
reported to display a marked increase in PHF tau levels, as well as cognitive impairments, in the water maze test [27, 28]. In the open field test, however, rTg4510 mice exhibited no delay in gait initiation when compared to their WT littermates (Fig. 2K) (Latency to gait initiation [in sec]: WT, $1.8 \pm 0.5 ; \mathrm{rTg} 4510,2.0 \pm 0.4 ; \mathrm{p}=0.67$ ). This suggested that the GIF phenotype in JNPL3 mice likely arose from Prion-promoter-driven expression of P301L mutant tau in hindbrain areas. Therefore, we performed immunohistochemistry for highly phosphorylated PHF tau in JNPL3 brains using an antiPHF tau antibody, AT8. PHF tau was barely detectable in sagittal brain sections of WT mice, while high levels of PHF tau were observed in brainstem areas associated with locomotor initiation (Fig. 4A, B). Quantitative analysis of immunolabeling data revealed that the fluorescence intensity of PHF tau was significantly higher in the reticular formation of JNPL3 mice compared to their WT controls (Fig. 4C) (PHF tau-positive area [\%]: WT, 0.0083 \pm 0.0048 ; JNPL3, 0.14 \pm 0.027 ; $\mathrm{p}=0.0091$; intensity of PHF tau [arbitrary units, a.u.]: WT, 29.47 \pm 3.23 ; JNPL3, 55.55 $\pm 2.72 ; \mathrm{p}=0.0035)$. As these brainstem areas are closely associated with locomotor initiation, these results suggested that GIF in JNPL3 mice may be caused by dysfunctional brainstem networks.

\section{DISCUSSION}

Impaired cognitive and motor function has been described in various genetic animal models of neurodegenerative diseases including tauopathies [1]. The usefulness of mouse models depends on reproducibility and recapitulation of the human phenotype [29]. However, subtle behavioral alterations in mouse models may miss detection because of poor sensitivity of behavioral tests and inaccuracy of monitoring devices. These factors may limit reproducibility, validity of preclinical research, and opportunities to investigate neural mechanisms and potential therapeutic interventions for human behavioral symptoms. Therefore, it is crucial to understand the detailed behavioral characteristics of mouse models for better clinical translation of basic research findings.

To extend previous findings of progressive motor disturbances in JNPL3 mice [11], here we examined whether JNPL3 mice exhibited novel behavioral phenotypes that recapitulated the motor deficits observed in human tauopathies. Using a battery of motor behavioral tests and high-speed video tracking system, we initially observed that JNPL3 mice displayed severe interlimb discoordination in trotting gait patterns (Fig. 1). The disrupted trotting pattern and longer interval between diagonal paw contacts in JNPL3 mice are likely due to weakened paw strength and hindlimb paralysis, with age- and gene-dose-dependent development of NFT [11].

Notably, we discovered that JNPL3 mice exhibited a marked delay in gait initiation (Fig. 2) that mirrors GIF syndrome in human patients with some forms of tauopathies such as Parkinson's disease, progressive supranuclear palsy, and corticobasal degeneration $[12,13]$. This gait freezing in JNPL3 mice was not a result of heightened anxiety because they exhibited comparable anxiety levels to those of WT controls in the elevated plus maze test (Fig. 3 ). In addition, eye irritation has been observed in JNPL3 homozygous mice, possibly due to the presence of the Pde $6 \mathrm{~b}^{\text {rd1 }}$ retinal degeneration allele [11]. However, the retinal degeneration mutation $(\mathrm{rd} / \mathrm{rd})$ is unlikely to cause gait freezing in JNPL3 mice because homozygous mice with the mutation perform normally on sensorimotor tasks such as the rotarod test [30]. Furthermore, we did not detect any signs of severe visual abnormalities in JNPL3 mice that may have led to limited activity or bumps into the walls of the arena during the open field test.

In this study, we observed a marked increase in fluorescence intensity of highly phosphorylated PHF tau in JNPL3 mice, specifically in nuclei involved in locomotor initiation, including the brainstem nuclei, and reticular formation (Fig. 4). The reticular formation sends glutamatergic projections to spinal central pattern generators [31]. Emerging evidence suggests that dysfunction of the pontomedullary reticular formation is implicated in the causation of gait freezing [32]. Furthermore, gait freezing has been proposed to arise from disconnection of the frontal lobes from subcortical and brainstem nuclei [33]. Collectively, our findings support previous evidence and strongly suggest that GIF in JNPL3 mice may be caused by aberrant synaptic function in brainstem networks. It will be of interest to examine whether optogenetic and/or pharmacological manipulations of neural circuits associated with locomotor initiation alters the GIF phenotype in JNPL3 mice. Indeed, recent clinical studies have reported beneficial effects of the dopamine agonist ropinirole, noradrenaline precursor L-threo-3,4-dihydroxyphenylserine, and subthalamic deep-brain stimulation on gait freezing in human patients [14, 23, 24, 34].

It should be noted that P301L-mutant tau may have beneficial effects in young adult JNPL3 mice, even though the mutation ultimately results in loss of motor neurons, paralysis, and death between 7 and 12 months $[11,35]$. At 5 7 months of age, JNPL3 mice were superior to control animals in the rotarod task, balance beam, and coat hanger tests [35]. However, only hemizygous JNPL3 mice that express transgenic tau at levels equivalent to endogenous tau were used in the behavioral experiments. Therefore, it appears that modest tau or mutant tau expression in JNPL3 mice may provide certain benefits for motor performance before the onset of neurodegeneration.

In summary, we demonstrate for the first time that JNPL3 human P301L-mutant tau mice display gait freezing that recapitu- 
lates the GIF phenotype in human patients. Our findings suggest that gait freezing in JNPL3 mice arises from network dysfunction of the hindbrain motor system. This study underscores the value of this mouse model as a tool to study the cellular mechanisms and potential therapeutics for human GIF syndrome.

\section{ACKNOWLEDGEMENTS}

The authors thank Ms. Na Young Do for the schematic illustration describing the findings of this study. This work was supported by the KBRI basic research program (18-BR-01-01; L.K.J.) and a National Research Foundation of Korea grant (NRF2017M3C7A1048086; L.K.J.) funded by Ministry of Science and ICT, the KIST Institutional Program (Project No. 2N4368018-045; L.K.J. and A.N.P.), and KBSI R\&D program (Project No. D37700; L.K.J.). The authors declare no competing financial interests.

\section{SUPPLEMENT}

Video S1. High-speed video recording of limb coordination in WT and JNPL3 mice.

Video S2. Latency to gait initiation in 12-month-old WT and JNPL3 mice.

\section{REFERENCES}

1. Dawson TM, Golde TE, Lagier-Tourenne C (2018) Animal models of neurodegenerative diseases. Nat Neurosci 21:13701379.

2. Brion JP (1998) The role of neurofibrillary tangles in Alzheimer disease. Acta Neurol Belg 98:165-174.

3. Love S, Bridges LR, Case CP (1995) Neurofibrillary tangles in Niemann-Pick disease type C. Brain 118:119-129.

4. Dickson DW (1999) Neuropathologic differentiation of progressive supranuclear palsy and corticobasal degeneration. J Neurol 246 Suppl 2:II6-II15.

5. Borroni B, Alberici A, Buratti E (2019) Review: Molecular pathology of frontotemporal lobar degenerations. Neuropathol Appl Neurobiol 45:41-57.

6. Hutton M, Lendon CL, Rizzu P, Baker M, Froelich S, Houlden H, Pickering-Brown S, Chakraverty S, Isaacs A, Grover A, Hackett J, Adamson J, Lincoln S, Dickson D, Davies P, Petersen RC, Stevens M, de Graaff E, Wauters E, van Baren J, Hillebrand M, Joosse M, Kwon JM, Nowotny P, Che LK, Norton J, Morris JC, Reed LA, Trojanowski J, Basun H, Lannfelt L, Neystat M, Fahn S, Dark F, Tannenberg T, Dodd PR, Hayward N,
Kwok JB, Schofield PR, Andreadis A, Snowden J, Craufurd D, Neary D, Owen F, Oostra BA, Hardy J, Goate A, van Swieten J, Mann D, Lynch T, Heutink P (1998) Association of missense and 5 -splice-site mutations in tau with the inherited dementia FTDP-17. Nature 393:702-705.

7. Dickson DW (1997) Neurodegenerative diseases with cytoskeletal pathology: a biochemical classification. Ann Neurol 42:541-544.

8. Kolarova M, García-Sierra F, Bartos A, Ricny J, Ripova D (2012) Structure and pathology of tau protein in Alzheimer disease. Int J Alzheimers Dis 2012:731526.

9. Lepeta K, Lourenco MV, Schweitzer BC, Martino Adami PV, Banerjee P, Catuara-Solarz S, de La Fuente Revenga M, Guillem AM, Haidar M, Ijomone OM, Nadorp B, Qi L, Perera ND, Refsgaard LK, Reid KM, Sabbar M, Sahoo A, Schaefer N, Sheean RK, Suska A, Verma R, Vicidomini C, Wright D, Zhang XD, Seidenbecher C (2016) Synaptopathies: synaptic dysfunction in neurological disorders - a review from students to students. J Neurochem 138:785-805.

10. Levenga J, Krishnamurthy P, Rajamohamedsait H, Wong H, Franke TF, Cain P, Sigurdsson EM, Hoeffer CA (2013) Tau pathology induces loss of GABAergic interneurons leading to altered synaptic plasticity and behavioral impairments. Acta Neuropathol Commun 1:34.

11. Lewis J, McGowan E, Rockwood J, Melrose H, Nacharaju P, Van Slegtenhorst M, Gwinn-Hardy K, Paul Murphy M, Baker M, Yu X, Duff K, Hardy J, Corral A, Lin WL, Yen SH, Dickson DW, Davies P, Hutton M (2000) Neurofibrillary tangles, amyotrophy and progressive motor disturbance in mice expressing mutant (P301L) tau protein. Nat Genet 25:402-405.

12. Factor SA, Jennings DL, Molho ES, Marek KL (2002) The natural history of the syndrome of primary progressive freezing gait. Arch Neurol 59:1778-1783.

13. Taskapilioglu O, Karli N, Erer S, Zarifoglu M, Bakar M, Turan F (2009) Primary gait ignition disorder: report of three cases. Neurol Sci 30:333-337.

14. Cohen-Oram AN, Stewart JT, Bero K, Hoffmann MW (2014) Treatment of gait ignition failure with ropinirole. J Mov Disord 7:95-98.

15. Yeo DK, Kwon KY (2017) Late onset aqueductal stenosisassociated hydrocephalus mimicking pure akinesia with gait freezing. Can J Neurol Sci 44:212-214.

16. Yoon SY, Lee SC, Kim NY, An YS, Kim YW (2017) Brain metabolism in patients with freezing of gait after hypoxicischemic brain injury: a pilot study. Medicine (Baltimore) 96:e8212.

17. Bloem BR, Hausdorff JM, Visser JE, Giladi N (2004) Falls and 
freezing of gait in Parkinson's disease: a review of two interconnected, episodic phenomena. Mov Disord 19:871-884.

18. Osaki Y, Morita Y, Miyamoto Y, Furuta K, Furuya H (2017) Freezing of gait is an early clinical feature of progressive supranuclear palsy. Neurol Clin Neurosci 5:86-90.

19. Abe K, Hashimoto T, Tamaru F, Ueno E, Yanagisawa N (1995) Analysis of gait disturbance in a patient with corticobasal degeneration. Rinsho Shinkeigaku 35:153-157.

20. Tardieu S, Becker H, Micallef J, Blin O (2003) Treatment of non-dopamine-dependent signs in parkinsonian syndromes: evaluation and results. Rev Neurol (Paris) 159:3S87-3S92.

21. Hwang HW, Lee SH, Lyoo CH, Lee MS (2017) Paroxysmal freezing of gait in a patient with mesial frontal transient ischemic attacks. BMC Neurol 17:122.

22. Atchison PR, Thompson PD, Frackowiak RS, Marsden CD (1993) The syndrome of gait ignition failure: a report of six cases. Mov Disord 8:285-292.

23. Fukada K, Endo T, Yokoe M, Hamasaki T, Hazama T, Sakoda S (2013) L-threo-3,4-dihydroxyphenylserine (L-DOPS) coadministered with entacapone improves freezing of gait in Parkinson's disease. Med Hypotheses 80:209-212.

24. Tohgi H,Abe T, Takahashi S (1993) The effects of L-threo-3,4dihydroxyphenylserine on the total norepinephrine and dopamine concentrations in the cerebrospinal fluid and freezing gait in parkinsonian patients. J Neural Transm Park Dis Dement Sect 5:27-34.

25. Lalonde R, Thifault S (1994) Absence of an association between motor coordination and spatial orientation in lurcher mutant mice. Behav Genet 24:497-501.

26. Bouwknecht JA, Spiga F, Staub DR, Hale MW, Shekhar A, Lowry CA (2007) Differential effects of exposure to lowlight or high-light open-field on anxiety-related behaviors: relationship to c-Fos expression in serotonergic and nonserotonergic neurons in the dorsal raphe nucleus. Brain Res Bull 72:32-43.

27. Ramsden M, Kotilinek L, Forster C, Paulson J, McGowan E, SantaCruz K, Guimaraes A, Yue M, Lewis J, Carlson G, Hutton M, Ashe KH (2005) Age-dependent neurofibrillary tangle formation, neuron loss, and memory impairment in a mouse model of human tauopathy (P301L). J Neurosci 25:1063710647.

28. Santacruz K, Lewis J, Spires T, Paulson J, Kotilinek L, Ingelsson M, Guimaraes A, DeTure M, Ramsden M, McGowan E, Forster C, Yue M, Orne J, Janus C, Mariash A, Kuskowski M, Hyman B, Hutton M, Ashe KH (2005) Tau suppression in a neurodegenerative mouse model improves memory function. Science 309:476-481

29. Justice MJ, Dhillon P (2016) Using the mouse to model human disease: increasing validity and reproducibility. Dis Model Mech 9:101-103.

30. Garcia MF, Gordon MN, Hutton M, Lewis J, McGowan E, Dickey CA, Morgan D, Arendash GW (2004) The retinal degeneration (rd) gene seriously impairs spatial cognitive performance in normal and Alzheimer's transgenic mice. Neuroreport 15:73-77.

31. Jordan LM, Liu J, Hedlund PB, Akay T, Pearson KG (2008) Descending command systems for the initiation of locomotion in mammals. Brain Res Brain Res Rev 57:183-191.

32. Nonnekes J, Geurts AC, Nijhuis LB, van Geel K, Snijders AH, Bloem BR, Weerdesteyn V (2014) Reduced StartReact effect and freezing of gait in Parkinson's disease: two of a kind? J Neurol 261:943-950.

33. Nutt JG, Bloem BR, Giladi N, Hallett M, Horak FB, Nieuwboer A (2011) Freezing of gait: moving forward on a mysterious clinical phenomenon. Lancet Neurol 10:734-744.

34. Varriale P, Collomb-Clerc A, Van Hamme A, Perrochon A, Kemoun G, Sorrentino G, George N, Lau B, Karachi C, Welter ML (2018) Decreasing subthalamic deep brain stimulation frequency reverses cognitive interference during gait initiation in Parkinson's disease. Clin Neurophysiol 129:24822491.

35. Morgan D, Munireddy S, Alamed J, DeLeon J, Diamond DM, Bickford P, Hutton M, Lewis J, McGowan E, Gordon MN (2008) Apparent behavioral benefits of tau overexpression in P301L tau transgenic mice. J Alzheimers Dis 15:605-614. 